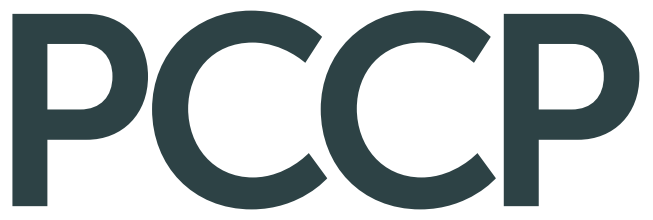

Physical Chemistry Chemical Physics www.rsc.org/pccp

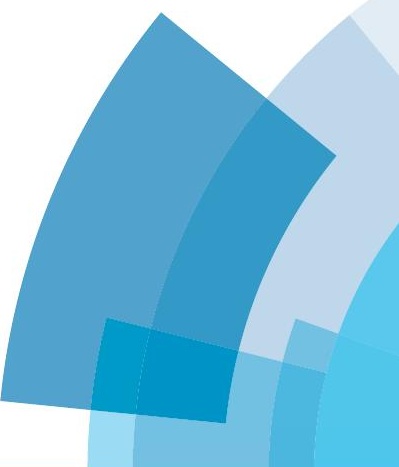

ISSN 1463-9076

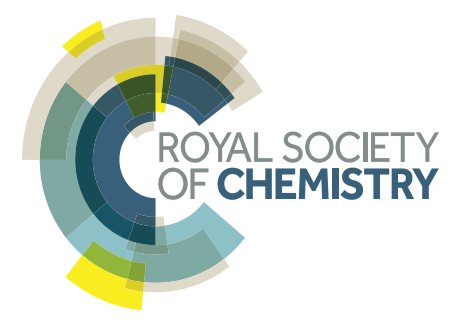




\section{Two equivalent methyl internal rotations in 2,5-dimethylthiophene investigated by microwave spectroscopy $\dagger$}

2015, 17, 32111

Received 17th June 2015,

Accepted 26th September 2015

DOI: $10.1039 / c 5 c p 03513 a$

www.rsc.org/pccp

The microwave spectrum of 2,5-dimethylthiophene, a sulfur-containing five-membered ring with two conjugated double bonds, was recorded in the frequency range from 2 to $40 \mathrm{GHz}$ using molecular beam Fourier transform technique. Highly accurate molecular parameters were determined. A labeling scheme for the group $G_{36}$ written as the semi-direct product $\left(C_{3}^{1} \times C_{3}^{1}\right) \rtimes C_{2 v}$ was introduced.

\section{Introduction}

Monomethyl derivatives of unsaturated heterocyclic five-membered rings have been widely studied by microwave spectroscopy. The barriers to internal rotation of the methyl group cover a wide range from low barriers such as in 2-methylthiazole (1), ${ }^{1} \mathrm{~N}$-methylpyrrole $(2){ }^{2}$ and 4-methylisothiazole $(3)^{3}$ to intermediate barriers like in 4-methyloxazole (4), ${ }^{4}$ 2-methylfuran (5), ${ }^{5}$ and 3-methylfuran (6), ${ }^{6}$ as well as 2-methylthiophene $(7)^{7}$ and 3-methylthiophene (8), ${ }^{8}$ as summarized in Fig. 1.

To the best of our knowledge, no microwave investigations on the rotational spectra of dimethyl substituted unsaturated heterocyclic five-membered rings are available. Here, we present our studies on 2,5-dimethylthiophene (DMTP) aimed at determining the torsional barriers of two equivalent methyl tops. A comparison of the experimental results with state of the art quantum chemical calculations (QCCs) will also be discussed.

\section{Quantum chemical calculations}

In DMTP, two conjugated double bonds force the thiophene frame to planarity, and thus only one conformer is possible. ${ }^{9}$ However, the orientations of the two methyl groups in the equilibrium, which determine the molecular symmetry $\left(C_{2 \mathrm{v}}, C_{2}\right.$, or $\left.C_{\mathrm{s}}\right)$, remain

\footnotetext{
${ }^{a}$ Institute of Physical Chemistry, RWTH Aachen University, Landoltweg 2, D-52074 Aachen, Germany. E-mail:vinh.van@rwth-aachen.de

${ }^{b}$ Laboratoire Interuniversitaire des Systèmes Atmosphériques (LISA), UMR 7583 (CNRS/Univ. Paris Est \& Paris Diderot), Université Paris Est, 61 avenue du Général de Gaulle, F-94010 Créteil cedex, France

$\dagger$ Electronic supplementary information (ESI) available. See DOI: 10.1039/c5cp03513a
}
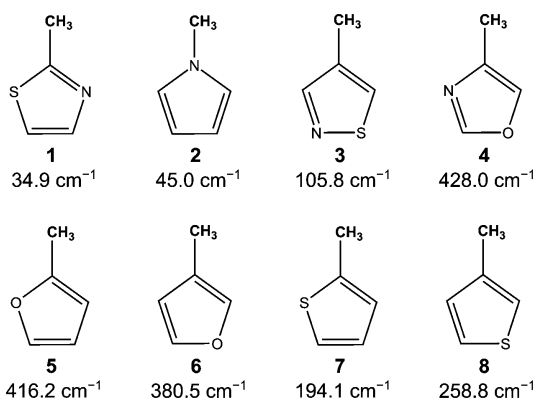

Fig. 1 Barriers to internal rotation of some monomethyl unsaturated heterocyclic five-membered rings.

questionable. In a previous electron diffraction study, ${ }^{10}$ one of the $\mathrm{C}-\mathrm{H}$ bonds of the methyl groups is cis with respect to the S-C bond, which is in contrast to QCCs at the HF/3-21G(d) level. In our work, much higher levels of theory are applied to study this observation.

Potential energy curves were obtained by varying the dihedral angle $\varphi_{1}=\angle\left(\mathrm{S}_{15}, \mathrm{C}_{12}, \mathrm{C}_{10}, \mathrm{H}_{4}\right)$ in a step width of $1^{\circ}$, while all other geometry parameters were optimized (for atom numbers see Fig. 2). Calculations were performed using the GAUSSIAN 09 package $^{11}$ with the MP2, B3LYP, and M06-2X methods and various basis sets. Three potential energy curves calculated with the cc-pVTZ basis set are shown in Fig. S-F1 in the ESI. $\dagger$ The $V_{3}$ potentials possess negligible contributions of higher order terms, which can be clearly recognized through the Fourier coefficients indicated in Table S1 also in the ESI. $\dagger$ The energy minima show a $C_{2 \mathrm{v}}$ symmetry, where a hydrogen atom of each methyl group is antiperiplanar to the sulfur atom. The two methyl groups are thus equivalent. Full geometry optimizations and harmonic frequency calculations yielded similar results (see Fig. 2), which are also in agreement with the calculations given in ref. 10. The Cartesian coordinates are available in Table S2 (ESI $\dagger$ ).

To study the coupling between the two methyl tops, a potential energy surface (PES) was calculated at the MP2/6-311++G(d,p) level of theory by varying the dihedral angles $\varphi_{1}$ and $\varphi_{2}=\angle\left(\mathrm{S}_{15}, \mathrm{C}_{11}, \mathrm{C}_{9}, \mathrm{H}_{1}\right)$ in a step width of $10^{\circ}$, as given in Fig. S-F2 (ESI $\dagger$ ). The corresponding 


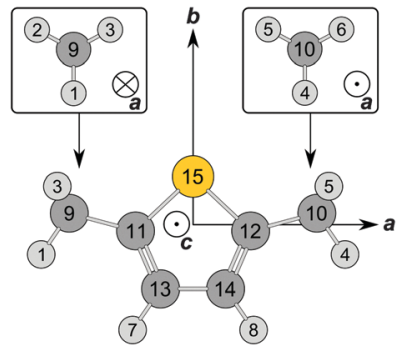

Fig. 2 The molecular geometry of DMTP calculated at the MP2/cc-pVTZ level of theory given in its principal axes of inertia. The direction of the axes into and out of the paper plane is indicated by an encircled cross or dot, respectively.

energies were parameterized with a two-dimensional Fourier expansion based on terms representing the correct symmetry; the Fourier coefficients are listed in Table S3 (ESI $\dagger$ ). No significant coupling between the two tops could be observed. We found a similar situation for the PES calculated at the B3LYP/6-311++G(d,p) level (visualized in Fig. S-F3, ESI $\dagger$ ). However, the energy minima are broader; the contribution of cross terms is smaller, as can also be recognized in Table S3 (ESI $\dagger$ ).

Geometry optimizations to a first order transition state of one methyl group using the Berny algorithm ${ }^{12}$ at various levels of theory were used to calculate the barriers to internal rotation. The calculated rotational constants, angles between an internal rotor axis and the principal axes, as well as the $V_{3}$ potentials are summarized in Table S4 in the ESI. $\dagger$ The angle $\angle\left(i_{2}, a\right)$ for the second rotor is $\pi-\angle\left(i_{1}, a\right)$, whereas $\angle\left(i_{1}, n\right)=\angle\left(i_{2}, n\right)$ for $n=b, c$. We noticed that the geometry optimizations and harmonic frequency calculations at the MP2/6-311++G(d,p) level yielded one imaginary vibrational mode, which is a bending of the planar thiophene frame. Stating stable planar ring systems as unstable is a well-known behavior found at this level of theory, which has been reported for benzene and arenes. ${ }^{13}$

\section{Group theory}

\section{Symmetry labels}

DMTP has a $C_{2 \mathrm{v}}$ frame symmetry and two equivalent methyl rotors. It is well known that the molecular symmetry group of such a system is $\mathrm{G}_{36}$ with the character table given in Table 1 . There are different symmetry labels in use which arise from different product decompositions of $\mathrm{G}_{36}$. Probably, the earliest method is based on the direct product $C_{3 \mathrm{v}}^{-} \times C_{3 \mathrm{v}}^{+}$, and the symmetry species of $\mathrm{G}_{36}$ are labeled with the labels of both subgroups $C_{3 \mathrm{v}}^{-}$and $C_{3 \mathrm{v}}^{+14}$ as given in column $\mathrm{S} 3$ of Table 1 . Using the permutation-inversion group formalism, Bunker and Jensen ${ }^{15}$ derived a character table of acetone, where the different species are denoted by their degeneracy letter A, E, G and a running index (shown in column S2 of Table 1).

We prefer a third labeling scheme (column S1), for which $\mathrm{G}_{36}$ is written as the semi-direct product $\left(C_{3}^{\mathrm{I}} \times C_{3}^{\mathrm{I}}\right) \triangleleft C_{2 \mathrm{v}}$, as reported by Ezra. ${ }^{16}$ The theoretical background was described in detail also by Altmann. ${ }^{17}$ The direct product $C_{3}^{\mathrm{I}} \times C_{3}^{\mathrm{I}}$ of the two intrinsic (superscript I) $C_{3}$ groups of the internal rotors, which is an invariant subgroup of $\mathrm{G}_{36}$, decomposes into four
Table 1 Character table of the molecular symmetry group $G_{36}$ of DMTP

\begin{tabular}{|c|c|c|c|c|c|c|c|c|c|c|c|c|}
\hline $\mathrm{G}_{36}{ }^{a}$ & & & $E$ & $a^{-1}$ & $a b$ & & $P$ & & & $a Q$ & & \\
\hline Equiv. & ot. ${ }^{b}$ & & $R^{0}$ & $R^{0}$ & $R^{0}$ & $R^{0}$ & $R_{b}^{\pi}$ & $R_{b}^{\pi}$ & $\underline{R_{a}^{\pi}}$ & $\underline{R_{a}^{\pi}}$ & $\underline{R_{c}^{\pi}}$ & \\
\hline $\mathrm{S} 1^{c}$ & $\mathrm{~S} 2^{d}$ & $\mathrm{~S} 3^{e}$ & $f_{1}$ & 4 & 2 & 2 & 3 & 6 & 3 & 6 & 9 & $\mathrm{wt}^{g}$ \\
\hline 0) $\cdot A_{1}$ & $\mathrm{~A}_{1}$ & $\mathrm{~A}_{1} \mathrm{~A}_{1}$ & 1 & 1 & 1 & 1 & 1 & 1 & 1 & 1 & 1 & 36 \\
\hline 0) $\cdot \mathrm{B}_{1}$ & $\mathrm{~A}_{2}$ & $\mathrm{~A}_{2} \mathrm{~A}_{1}$ & 1 & 1 & 1 & 1 & -1 & -1 & 1 & 1 & -1 & 28 \\
\hline 00$) \cdot A_{2}$ & $\mathrm{~A}_{3}$ & $\mathrm{~A}_{1} \mathrm{~A}_{2}$ & 1 & 1 & 1 & 1 & 1 & 1 & -1 & -1 & -1 & 36 \\
\hline$(00) \cdot B_{2}$ & $\mathrm{~A}_{4}$ & $\mathrm{~A}_{2} \mathrm{~A}_{2}$ & 1 & 1 & 1 & 1 & -1 & -1 & -1 & -1 & 1 & 28 \\
\hline$(12) \cdot A^{\prime}$ & $\mathrm{E}_{1}$ & $\mathrm{E} \mathrm{A}_{1}$ & 2 & -1 & 2 & -1 & 0 & 0 & 2 & -1 & 0 & 16 \\
\hline$(12) \cdot A^{\prime \prime}$ & $E_{2}$ & $\mathrm{E} \mathrm{A}_{2}$ & 2 & -1 & 2 & -1 & 0 & 0 & -2 & 1 & 0 & 16 \\
\hline$(11) \cdot A$ & $\mathrm{E}_{3}$ & $\mathrm{~A}_{1} \mathrm{E}$ & 2 & -1 & -1 & 2 & 2 & -1 & 0 & 0 & 0 & 20 \\
\hline$(11) \cdot \mathrm{B}$ & $\mathrm{E}_{4}$ & $\mathrm{~A}_{2} \mathrm{E}$ & 2 & -1 & -1 & 2 & -2 & 1 & 0 & 0 & 0 & 12 \\
\hline$(01) \cdot A$ & G & EE & 4 & 1 & -2 & -2 & 0 & 0 & 0 & 0 & 0 & 64 \\
\hline
\end{tabular}

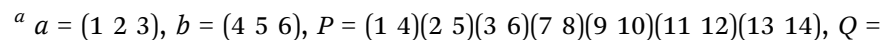
(1 4)(2 6)(3 5)(7 8)(9 10)(11 12)(13 14)* $R=\left(\begin{array}{l}2 \\ 2\end{array}\right)\left(\begin{array}{l}5 \\ \text { 6 }\end{array}\right)^{*}$, for atom numbers see Fig. $2 .{ }^{b}$ Equivalent rotations of the four-group. ${ }^{c}$ Symmetry labels based on the semi-direct product $\left(C_{3}^{\mathrm{I}} \times C_{3}^{\mathrm{I}}\right) \succ C_{2 \mathrm{v}}$, see ref. 16 . ${ }^{d}$ Symmetry labels according to ref. $15 .{ }^{e}$ Symmetry labels based on the direct product $C_{3 \mathrm{v}}^{-} \times C_{3 \mathrm{v}}^{+}$, see ref. $14 .{ }^{f}$ Row between S3 and wt: number of elements in the respective class. ${ }^{g}$ Spin statistical weight.

Table 2 Orbits of the species of $\left(C_{3}^{1} \times C_{3}^{1}\right)$ under $C_{2 v}$

\begin{tabular}{ll}
\hline Orbit $\left(\sigma_{1}, \sigma_{2}\right)$ & Little co-set \\
\hline$(00)$ & $C_{2 \mathrm{v}}$ \\
$(11),(22)$ & $C_{2}$ \\
$(12),(21)$ & $C_{\mathrm{s}}$ \\
$(01),(10),(02),(20)$ & $C_{1}$
\end{tabular}

orbits under $C_{2 \mathrm{v}}$ (Table 2). One representative of each orbit forms the first part of the symmetry label, e.g. (01). The numbers $\sigma=0$, 1,2 represent the three symmetry species $A, E_{a}, E_{b}$, respectively, of the group $C_{3}$ and correspond to the transformation properties of the $C_{3}$-adapted planar rotor wave functions $\mathrm{e}^{i(3 k+\sigma) \varphi}$ with $k \in \mathbb{Z}$ and the torsional angle $\varphi$. Each orbit is associated with its little co-set. They form subgroups of $C_{2 \mathrm{v}}$. The symmetry species of the little co-sets provide the second part of our symmetry labels.

\section{Nuclear spin statistics}

The total wave function $\psi_{\mathrm{tot}}=\psi_{\mathrm{el}} \cdot \psi_{\mathrm{vib}} \cdot \psi_{\text {rotors }} \cdot \psi_{\mathrm{ns}}$ must obey the Pauli principle, i.e. it changes sign under an odd number of fermion permutations (here protons) and retains sign under an even number of permutations. It belongs thus either to the species $(00) \cdot A_{1}$ or $(00) \cdot A_{2}$. There are 256 nuclear spin functions arising from 8 protons in DMTP with the representation $\Gamma_{\mathrm{ns}}=36(00) \cdot \mathrm{A}_{1}+28(00) \cdot \mathrm{B}_{2}+10(12) \cdot \mathrm{A}^{\prime}+6(12) \cdot \mathrm{A}^{\prime \prime}+10(11) \cdot$ $\mathrm{A}+6(11) \cdot \mathrm{B}+32(01) \cdot \mathrm{A}$.

We determined the spin weights (column wt in Table 1) by counting the number of allowed total wave functions for $\Gamma_{\mathrm{el}}=\Gamma_{\mathrm{vib}}=(00) \cdot \mathrm{A}_{1}$.

\section{Selection rules}

The dipole moment vector in space fixed coordinates is invariant under pure permutation operations but changes sign under inversion. Therefore, it transforms as $\Gamma_{\mu}=(00) \cdot \mathrm{A}_{2}$. In the vibronic ground state, transitions between two rotorsional levels $i$ and $j$ are allowed, if $\Gamma_{i} \times \Gamma_{\mu} \times \Gamma_{j} \supset(00) \cdot \mathrm{A}_{1}$. The resulting four torsional components of two different b-type transitions, namely 
Table 3 Spin statistical weights of torsional components of allowed transitions of DMTP

\begin{tabular}{llll}
\hline ee $\leftrightarrow$ oo transitions & wt & eo $\leftrightarrow$ oe transitions & wt \\
\hline$(00) \cdot \mathrm{A}_{1} \leftrightarrow(00) \cdot \mathrm{A}_{2}$ & 36 & $(00) \cdot \mathrm{B}_{1} \leftrightarrow(00) \cdot \mathrm{B}_{2}$ & 28 \\
$(12) \cdot \mathrm{A}^{\prime} \leftrightarrow(12) \cdot \mathrm{A}^{\prime \prime}$ & 16 & $(12) \cdot \mathrm{A}^{\prime} \leftrightarrow(12) \cdot \mathrm{A}^{\prime \prime}$ & 16 \\
$(11) \cdot \mathrm{A} \leftrightarrow(11) \cdot \mathrm{A}$ & 20 & $(11) \cdot \mathrm{B} \leftrightarrow(11) \cdot \mathrm{B}$ & 12 \\
$(01) \cdot \mathrm{A} \leftrightarrow(01) \cdot \mathrm{A}$ & 64 & $(01) \cdot \mathrm{A} \leftrightarrow(01) \cdot \mathrm{A}$ & 64
\end{tabular}

$K_{\mathrm{a}} K_{\mathrm{c}}$ : ee $\leftrightarrow$ oo and eo $\leftrightarrow$ oe (e = even, o = odd) along with their spin statistical weights are given in Table 3 . They are determined by correlation with the molecular symmetry group $C_{2 \mathrm{v}}(\mathrm{M})$ of the hypothetical rigid DMTP. If the quantum numbers $K_{\mathrm{a}}$ and $K_{\mathrm{c}}$ are known, the torsional state can be labeled conveniently by the first part $\left(\sigma_{1}, \sigma_{2}\right)$ of the full symmetry label. We will use this abbreviated notation throughout this paper.

\section{Microwave spectroscopy}

\section{Experimental setup}

The rotational spectra were measured with two molecular beam Fourier transform microwave spectrometers covering the frequency ranges from 2 to $26.5 \mathrm{GHz}^{18}$ and from 26.5 to $40 \mathrm{GHz} .{ }^{19}$ DMTP was purchased from Alfa Aesar GmbH \& Co KG, Karlsruhe, Germany with a stated purity of over 98\%. Some drops of the liquid substance were put on a $5 \mathrm{~cm}$ piece of a pipe cleaner, which was placed inside a steel tube close to the nozzle. A helium stream at a pressure of approximately $200 \mathrm{kPa}$ flowed over the substance and the helium-substance mixture was expanded into the cavity.

\section{Spectral analysis}

The QCC results have shown that DMTP does not possess dipole moment components in $a$ - and $c$-direction. Only b-type transitions are thus expected, which is in agreement with the group theoretical results (see section Selection rules). In the first step, the b-type rigid-rotor spectrum was predicted using the rotational constants calculated at the MP2/cc-pVTZ level. Based on this prediction, a broadband scan in the frequency range from 8.5 to $12.0 \mathrm{GHz}$ was measured, where overlapping spectra in a step width of $0.25 \mathrm{MHz}$ were automatically recorded. In the next step, all lines were remeasured at higher resolution.

At the beginning, the transitions $2_{20} \leftarrow 2_{11}, 3_{21} \leftarrow 3_{12}$, and $4_{22} \leftarrow 4_{13}$ as well as $2_{21} \leftarrow 2_{12}$ and $3_{22} \leftarrow 3_{13}$ were considered. They were not the most intensive transitions in the scan but their positions could be predicted quite precisely. After these transitions had been assigned and fitted, further lines in the scan were added to the fit. Afterwards, transitions outside the scan region were also included. The results are given as Fit I in Table 4. A prediction considering the internal rotation of both methyl groups was then carried out using the program XIAM. ${ }^{20}$ The angles $\angle\left(i_{1}, n\right)$ and $\angle\left(i_{2}, n\right), n=a, b, c$, as well as the initial $V_{3}$ potential were taken from the values calculated at the MP2/cc-pVTZ level. Supported by the intensities from the spin statistical weights, the (01), (11), and (12) species in the scan were successfully assigned. The splittings were up to $0.5 \mathrm{GHz}$. Finally, further torsional transitions up to $J=7$ and $K_{a}=5$ were measured to increase the data set.
Table 4 Molecular parameters of DMTP obtained by the program XIAM. Only the $(00)$ species is fitted in Fit l, whereas all four species are taken into account in Fit II

\begin{tabular}{lllll}
\hline Par. $^{a}$ & Unit & Fit I & Fit II & Calc. $^{b}$ \\
\hline$A$ & $\mathrm{GHz}$ & $4.97224360(42)$ & $4.96500697(89)$ & 4.9414 \\
$B$ & $\mathrm{GHz}$ & $1.74696506(27)$ & $1.74694756(13)$ & 1.7426 \\
$C$ & $\mathrm{GHz}$ & $1.31337981(20)$ & $1.313336623(81)$ & 1.3094 \\
$\Delta_{\mathrm{J}}$ & $\mathrm{kHz}$ & $0.0684(28)$ & $0.0672(11)$ & 0.0630 \\
$\Delta_{\mathrm{JK}}$ & $\mathrm{kHz}$ & $0.2071(93)$ & $0.1579(37)$ & 0.171 \\
$\Delta_{\mathrm{K}}$ & $\mathrm{kHz}$ & $3.2158(98)$ & $0.9161(43)$ & 0.803 \\
$\delta_{\mathrm{J}}$ & $\mathrm{kHz}$ & $0.0196(14)$ & $0.01839(51)$ & 0.0171 \\
$\delta_{\mathrm{K}}$ & $\mathrm{kHz}$ & $0.136(23)$ & $0.1031(89)$ & 0.0390 \\
$V_{3}$ & $\mathrm{~cm}^{-1}$ & & $247.95594(30)$ & 241.2 \\
$I_{\alpha}$ & $\mathrm{u}{ }^{2}$ & & $3.16442(39)$ & \\
$\angle(i, a)$ & $\circ$ & & $14.5931(78)^{c}$ & 14.90 \\
$\angle(i, b)$ & $\circ$ & & $75.4069(78)$ & 75.10 \\
$\angle(i, c)$ & $\circ$ & & $90.00(\mathrm{fixed})$ & 89.96 \\
$F_{12}$ & $\mathrm{GHz}$ & & $-7.320(12)$ & \\
$D_{\text {pi2J }}$ & $\mathrm{kHz}$ & & $-2.78(25)$ & \\
$D_{\text {pi2K }}$ & $\mathrm{kHz}$ & & $-98.9(42)$ & \\
$D_{\text {pi2- }}$ & $\mathrm{kHz}$ & & $1.97(19)$ & \\
$\sigma^{d}$ & $\mathrm{kHz}$ & 2.6 & 1.9 & \\
$N^{e}$ & & 41 & 157 &
\end{tabular}

${ }^{a}$ All parameters refer to the principal axis system. Watson's A reduction and $\mathrm{I}^{\mathrm{r}}$ representation were used. A comparison with the fits using Watson's $S$ reduction is given in Table S6 in the ESI. ${ }^{b}$ At MP2/6-311++G(d,p) level. ${ }^{c}$ The angle $\angle\left(i_{2}, a\right)$ for the second rotor is derived from the relation $\pi-$ $\angle\left(i_{1}, a\right) .{ }^{d}$ Standard deviation of the fit. ${ }^{e}$ Number of lines.

The typical high resolution spectra of the four torsional components are visualized in Fig. 3. We notice that in many transitions, the (00) and the (01) species indicate additional small splittings of about $10 \mathrm{kHz}$. These splittings are probably due to spin-spin or spin-rotation coupling of the protons. However, for the (01) species, another explanation is possible. In $\mathrm{G}_{36}$ molecules, the (01) and the (10) species are degenerate. Under the influence of centrifugal distortion, this degeneracy could be lifted if rotations about axes other than $a, b$, and $c$ are considered. ${ }^{21}$ Nevertheless, since the splittings are small and no theory is available for spin-spin and spin-rotation couplings of molecules with 8 protons like DMTP, nor for the effect mentioned above, no support for this argument can be given. We note that

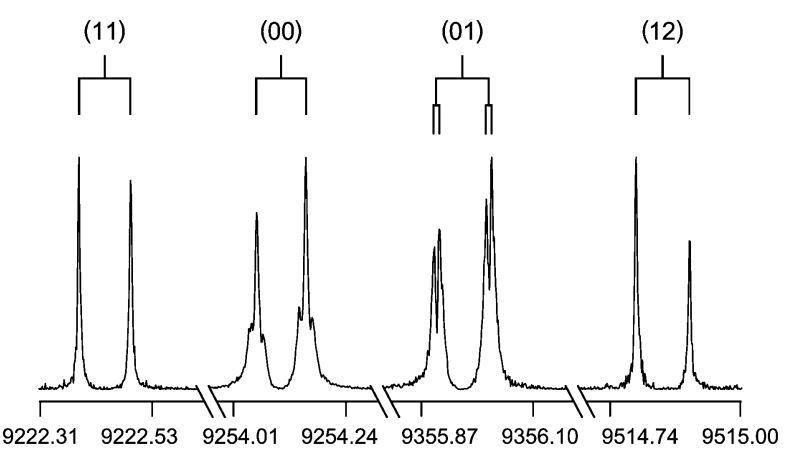

Fig. 3 Typical spectra of the four torsional species (00), (01), (11), and (12) of the $3_{21} \leftarrow 3_{12}$ transition of DMTP (in MHz). The experimental resolution is $2 \mathrm{kHz}$, the typical line width $10 \mathrm{kHz}(\mathrm{FWHH})$. The larger splittings indicated by brackets are due to the Doppler effect; the smaller brackets indicate splittings of unknown origin. For these spectra, 21-614 decays were co-added. The intensities are normalized. An energy level diagram to illustrate the splitting is given in Fig. S-F4 in the ESI. $\dagger$ 
though the PES in section Quantum chemical calculations shows negligible top-top interaction, the (11)-(12) splittings are not small. This observation is mainly related to the kinetic coupling.

\section{Results of the fits and discussion}

In total, 157 torsional components were fitted to a standard deviation of $1.9 \mathrm{kHz}$, within the measurement accuracy. The rotational and centrifugal distortion constants were well determined. The $V_{3}$ potential, the moment of inertia $I_{\alpha}$, the angles $\delta$ between the internal rotation axes and the principal $a$-axis, the kinetic coupling between the two rotors $F_{12}$, and three higher order parameters $D_{\text {pi2J }}, D_{\text {pi2K }}$, and $D_{\text {pi2- }}$ were also fitted as linear combinations of the two tops. The results are given as Fit II in Table 4; the frequency list is in Table S5 (ESI $\dagger$ ).

The experimental rotational constants were compared with those from QCCs. We found the best agreements with the method M06-2X, followed by MP2 and B3LYP, as shown in Table S4 (ESI $\dagger$ ). The smallest Pople basis set shows better agreement than bigger basis sets, which is probably the result of error compensation.

The calculated barriers to internal rotation vary in a wide range between $215.5 \mathrm{~cm}^{-1}$ and $405.2 \mathrm{~cm}^{-1}$, but they are still in the same order of magnitude as the experimental $V_{3}$ potential of $248.0 \mathrm{~cm}^{-1}$. The HF method overestimates the barrier by over $100 \mathrm{~cm}^{-1}$, whereas the MP2 method underestimates the barrier by up to $30 \mathrm{~cm}^{-1}$. We found that the Dunning basis sets yield better results than the Pople ones. The barrier of $248.4 \mathrm{~cm}^{-1}$ calculated at the MP2/6-311G(d,p) level matches the experimental value best.

The barriers of DMTP are closer to the value found for the mono-substituted molecule (8) rather than that of (7), which has a more similar structure (see Fig. 1). In molecules with other hetero atoms (oxygen or nitrogen) or with two hetero atoms, the barrier changes significantly. Our attempts to find out a rule to predict the methyl barriers in unsaturated heterocyclic five-membered rings failed. Unlike in alkyl acetates where the barrier remains largely invariant at approximately $100 \mathrm{~cm}^{-1}$, this value cannot be easily predicted when electronic effect exists, e.g. by the presence of double bonds. In unsaturated acetates, ${ }^{22,23} \pi$-electron conjugation, extending from the double bond to the ester group, is possible, which causes significantly different barriers.

For unsaturated rings with conjugated double bonds like DMTP, it is mostly probable that this electronic system influences the barrier heights. However, more investigations have to be performed to confirm this speculation and moving towards a more precise prediction of the methyl barriers.

\section{Conclusions}

The spectrum of DMTP recorded under molecular beam conditions was successfully assigned using a combination of FT microwave spectroscopy, group theory, and quantum chemistry. The rotational constants obtained by geometry optimizations at different levels of theory are in good agreement with the experimental values. A $C_{2 v}$ equilibrium structure was calculated, where one hydrogen atom of each methyl group is anti-periplanar to the sulfur atom, and the two methyl groups are thus equivalent. They undergo internal rotations with a barrier height of $247.95594(30) \mathrm{cm}^{-1}$, which is in reasonable agreement with the calculated barrier.

\section{Acknowledgements}

V.V. thanks the Fonds der Chemischen Industrie (FCI) for a PhD fellowship. Simulations were performed with computing resources granted by JARA-HPC from the RWTH Aachen University under the project jara0124.

\section{References}

1 J.-U. Grabow, H. Hartwig, N. Heineking, W. Jäger, H. Mäder, H. W. Nicolaisen and W. Stahl, J. Mol. Struct., 2002, 612, 349.

2 W. Arnold, H. Dreizler and H. D. Rudolph, Z. Naturforsch., A: Astrophys., Phys. Phys. Chem., 1968, 23, 301.

3 H.-W. Nicolaisen, J.-U. Grabow, N. Heineking and W. Stahl, Z. Naturforsch., A: Phys. Sci., 1991, 46, 635.

4 E. Fliege, Z. Naturforsch., A: Phys. Sci., 1990, 45, 911.

5 W. G. Norris and L. C. Krisher, J. Chem. Phys., 1969, 51, 403.

6 T. Ogata and K. Kozima, Bull. Chem. Soc. Jpn., 1971, 44, 2344.

7 N. M. Pozdeev, L. N. Gunderova and A. A. Shapkin, Opt. Spektrosk., 1970, 28, 254.

8 T. Ogata and K. Kozima, J. Mol. Spectrosc., 1972, 42, 38.

9 B. Bak, D. Christensen, L. Hansen-Nygaard and J. RastrupAndersen, J. Mol. Spectrosc., 1961, 7, 58.

10 M. Tanabe, N. Kuze, H. Fujiwara, H. Takeuchi and S. Konaka, J. Mol. Struct.: THEOCHEM, 1995, 372, 173.

11 M. J. Frisch, et al., Gaussian 09 (Revision A.02), Gaussian, Inc., Wallingford CT, 2009.

12 H. B. Schlegel, J. Comput. Chem., 1982, 3, 214.

13 D. Moran, A. C. Simmonett, F. E. Leach, W. D. Allen, P. v. R. Schleyer and H. F. Schaefer, J. Am. Chem. Soc., 2006, 128, 9342.

14 H. Dreizler, Z. Naturforsch., 1961, 16a, 1354.

15 P. R. Bunker and P. Jensen, Molecular Symmetry and Spectroscopy, NRC Research Press, Ottawa, Ontario, Canada, 2006.

16 G. S. Ezra, Symmetry Properties of Molecules, Lecture Notes in Chemistry 28, Springer-Verlag, Berlin, Heidelberg, New York, 1982.

17 S. L. Altmann, Induced Representations in Crystals and Molecules, Point, space and nonrigid molecule groups, Academic Press, London, New York, San Francisco, 1977.

18 J.-U. Grabow, W. Stahl and H. Dreizler, Rev. Sci. Instrum., 1996, 67, 4072.

19 I. Merke, W. Stahl and H. Dreizler, Z. Naturforsch., A: Phys. Sci., 1994, 49, 490.

20 H. Hartwig and H. Dreizler, Z. Naturforsch., A: Phys. Sci., 1996, 51, 923.

21 P. Jensen and P. R. Bunker, J. Mol. Spectrosc., 1994, 164, 315.

22 H. V. L. Nguyen, A. Jabri, V. Van and W. Stahl, J. Phys. Chem. A, 2014, 118, 12130.

23 H. V. L. Nguyen and W. Stahl, J. Mol. Spectrosc., 2010, 264, 120. 